\title{
Binary stars in moving groups
}

\section{Rebecca Azulay ${ }^{* \dagger}$}

Dpt. d'Astronomia i Astrofísica, Universitat de València, E-46100 Burjassot, València, Spain Max-Planck-Institut für Radioastronomie, D-53121 Bonn, Germany

E-mail: rebecca.azulayeuv.es

\section{J. C. Guirado, J. M. Marcaide}

Dpt. d'Astronomia i Astrofísica, Universitat de València, E-46100 Burjassot, València, Spain Observatori Astronòmic, Universitat de València, E-46980 Paterna, València, Spain

\section{Martí-Vidal}

Onsala Space Observatory, Chalmers University of Technology, SE-43992 Onsala, Sweden

\section{E. Ros}

Max-Planck-Institut für Radioastronomie, D-53121 Bonn, Germany

Observatori Astronòmic, Universitat de València, E-46980 Paterna, València, Spain

Dpt. d'Astronomia i Astrofísica, Universitat de València, E-46100 Burjassot, València, Spain

\begin{abstract}
Precise determination of dynamical masses of pre-main-sequence (PMS) stars is necessary to calibrate PMS stellar evolutionary models, whose predictions are in disagreement with measurements for masses below $1.2 \mathrm{M}_{\odot}$. Binary stars in young, nearby loose associations are particularly good candidates, since all members share a common age. We present phase-reference EVN observations of the binary system HD $160934 \mathrm{~A} / \mathrm{c}$, that belongs to the AB Doradus moving group, from which we have measured both the relative and absolute orbital motion. Accordingly, we obtained precise estimates of the mass of the components of this binary. Also we report on other PMS binary systems as EK Dra and AB Dor B.
\end{abstract}

12th European VLBI Network Symposium and Users Meeting

7-10 October 2014

Cagliari, Italy

\footnotetext{
* Speaker.

${ }^{\dagger}$ Guest student of the International Max Planck Research School for Astronomy and Astrophysics at the Universities of Bonn and Cologne
} 


\section{Introduction}

Stellar evolution models are used to derive fundamental parameters of stars, such as their mass. However, the predictions based on pre-main-sequence (PMS) models for stars with masses $<1.2 \mathrm{M}_{\odot}$ are questionable since the contrast with the observational data reveals some discrepancies. Calibrating stellar evolution models requires precise and independent measurements of luminosities and masses of these stars in order to compare them with the theoretical results. Some authors have noted the need to fit well the theoretical models for PMS stars [7, 15, 14], but there is a small number of these systems with masses $<1.2 \mathrm{M}_{\odot}$ observed so far [7].

Binary stars in young nearby moving groups are good candidates to increase the number of PMS stars with dynamically determined masses $[17,16]$. In particular, stars belonging to the AB Doradus moving group (AB Dor-MG) seem to be optimal for our study: AB Dor-MG is the closest one (its mean distance to the Sun is $30 \mathrm{pc}$ ), the estimated age is relatively accurate (50$70 \mathrm{Myr} ;[9,6]$ and it contains stars with significant emission at radio wavelengths [5, 2]. This last feature is essential, because it allows the use of radio interferometry techniques to obtain astrometric information. Following the list of AB Dor-MG members in [16], we initiated a VLA/VLBI program dedicated to monitor binary systems known to host low-mass companions, and which are likely to present radio emission. One of the stars included in our program is HD 160934.

\section{Stellar system and observations}

HD 160934 is a tertiary stellar system placed at a distance of $\sim 30 \mathrm{pc}$. We focus our study in the primary pair $\mathrm{A} / \mathrm{c}$, which components are separated $\sim 0.2^{\prime \prime}$. The component $\mathrm{B}$ is placed at a distance of $\sim 8.7^{\prime \prime}$ from the primary pair $[13,8]$. We observed this target in three different epochs: 2012 October 30, 2013 May 23, and 2014 March 5, using the European VLBI Network (EVN) at $5 \mathrm{GHz}$. Each observation lasted 10 hours with a data rate of $1024 \mathrm{Mbps}$ in dual polarization and was scheduled in phase-reference style, interleaving scans of the ICRF quasar J1746+6226 and the target star HD 160934. The cycle target-calibrator-target lasted about six minutes. Part of the results of this program has been already published in [2].

\section{Results}

\subsection{Maps of HD 160934}

The phase-referenced naturally-weighted images of the target are shown in Fig. 1. These maps reveal that HD 160934 is a relatively strong radio emitter, likely due to its high rotation rate ( $v \sin i=16.4 \mathrm{~km} \mathrm{~s}^{-1}$; [4]). From its $8.4 \mathrm{GHz}$ VLA flux and Hipparcos distance, we estimate the radio luminosity $L_{R}$ of this star, to be $0.27 \times 10^{15} \mathrm{erg} \mathrm{Hz}^{-1} \mathrm{~s}^{-1}$. This value is very similar to those of well-known radio star members of the AB Dor-MG, namely PW And, LO Peg, EK Dra, and AB Dor itself, the main star system of the AB Dor-MG.

The detection of the lower-brightness component in our VLBI maps (Fig. 1) is fortuitous, since our original plan was to monitor only the reflex motion of HD $160934 \mathrm{~A}$. This shows that radio activity is not a rare phenomenon in stars belonging to the AB Dor-MG, and, indeed, this is 

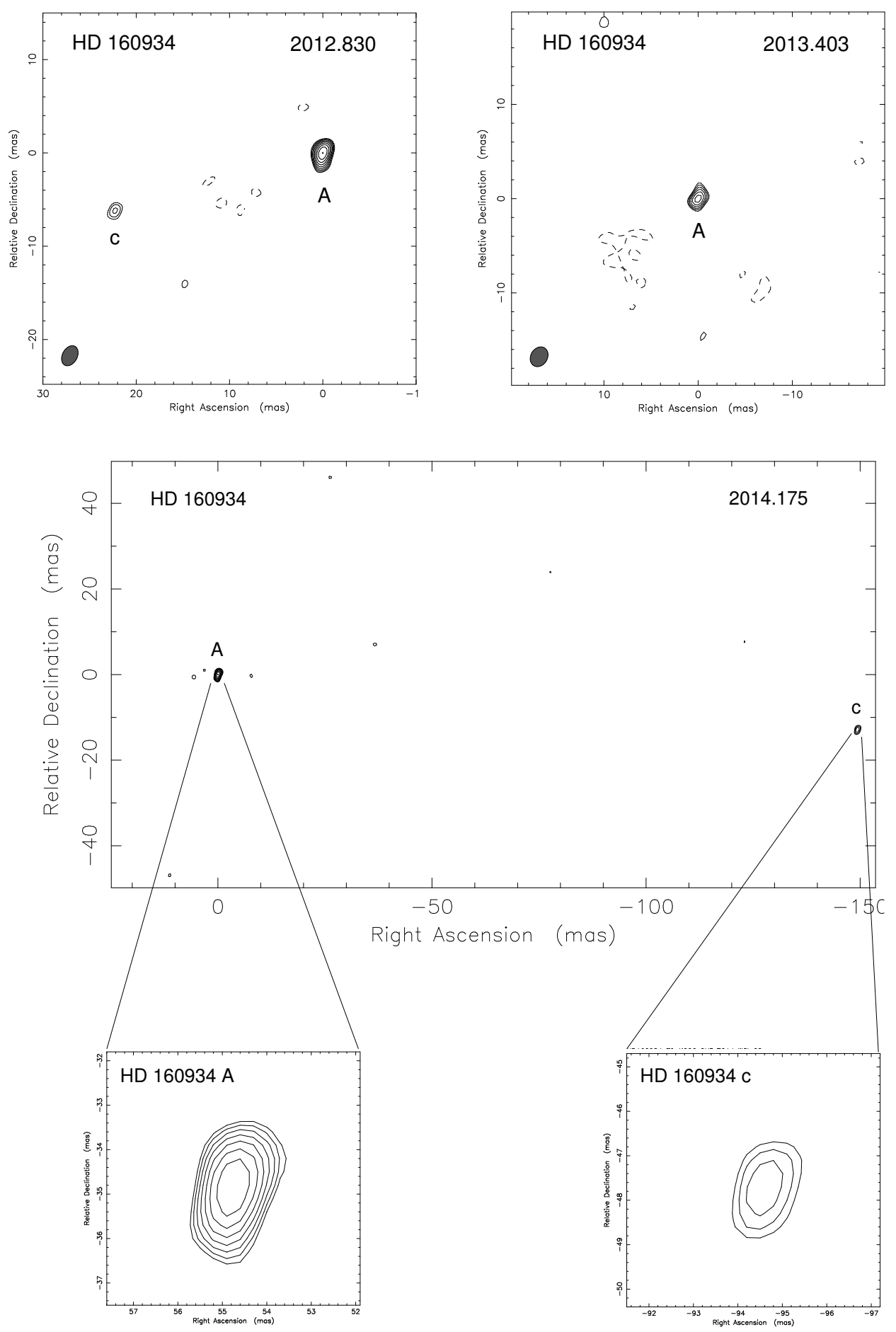

Figure 1: Clean maps of the binary HD 160934 at the three EVN epochs using the antennas at Effelsberg, Westerbork, Jodrell Bank, Onsala, Medicina, Noto, Torun, Yebes, Svetloe, Zelenchukskaya, Badary, Urumqi, and Shanghai. The lowest contour levels are, respectively, 0.04, 0.02, and $0.04 \mathrm{mJy} \mathrm{beam}^{-1}$. The scale factor between contiguous contours is $\sqrt{2}$. In all the maps we have centered at the origin the position of peak of brightness of HD 160934 A. Component $\mathrm{c}$ is not detected at epoch 2013.403. Orbital motion of $\mathrm{c}$ around A is evident from epochs 2012.830 and 2014.175 (Azulay et al., in preparation). 


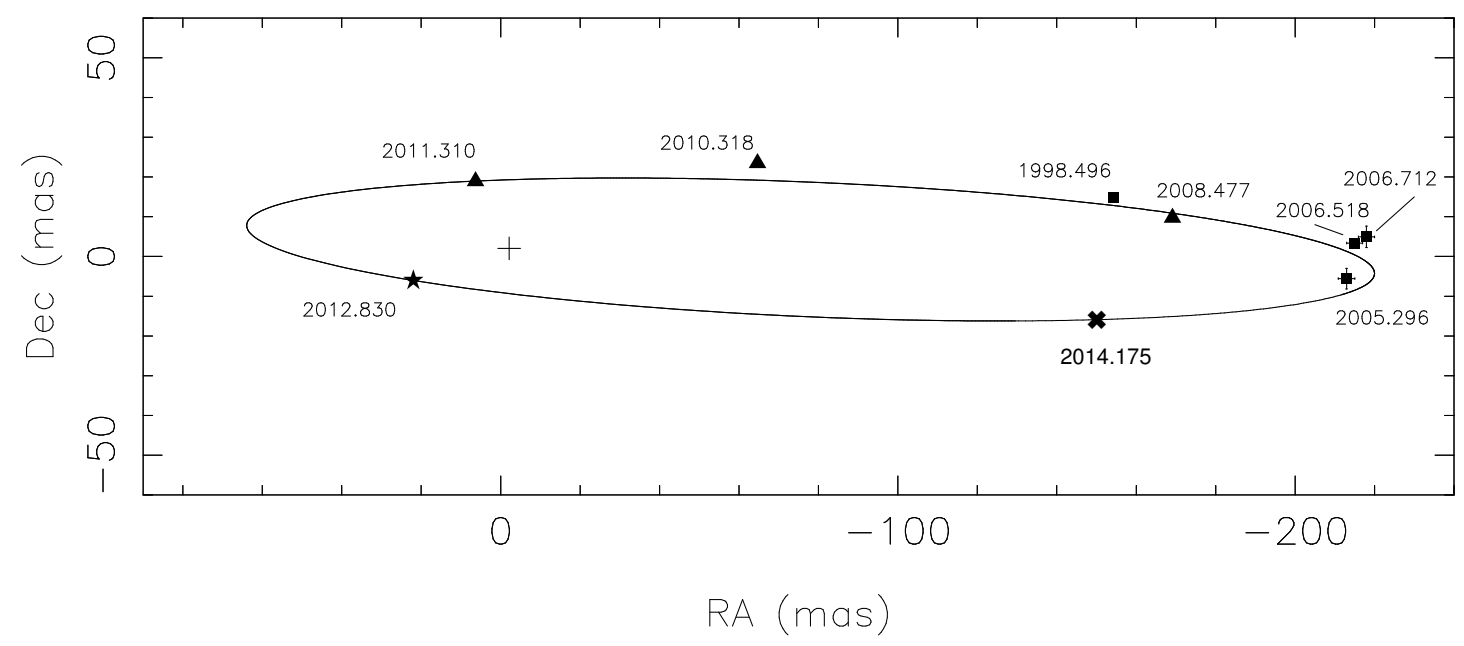

Figure 2: Orbit of the binary star HD 160934. HD $160934 \mathrm{~A}$ component is shown as a small cross at the origin. Each type of symbol corresponds to a different technique to measure the relative position of HD 160934 c, namely, infrared relative astrometry (squares; [8, 12]), masking interferometry (triangles; [3]), and VLBI (star symbol; [2], cross symbol; this work). Error bars are plotted but barely visible because of the size of the orbit.

not the first radio binary (i.e., both members of the binary are radio emitters) in this association (radio detections have been reported for $\mathrm{AB}$ Dor $\mathrm{Ba} / \mathrm{Bb}$ with masses down to $0.2 \mathrm{M}_{\odot}$, [1]).

\subsection{Determination of orbital parameters and masses}

The maps shown in Fig. 1 provide the absolute position of HD 160934 A with respect to the quasar at the three epochs. In addition, with the images of the stars at epochs 2012.830 and 2014.175, we can determine the relative position between A and c. Using these data together with NIR data available in the literature $[8,12,3]$, we determine simultaneously both the relative (Fig. 2 ) and the individual orbits of each pair. We used a least-square fit similar to that described in [5].

The sum of the masses of the system $\left(m_{1}+m_{2}\right)$ can be calculated from the Kepler's Third Law $\left(m_{1}+m_{2}=\left(a_{\text {rel }} \cdot d\right)^{3} / P^{2}\right)$, where $a_{\text {rel }}$ is the semimajor axis of the relative orbit $\left(^{\prime \prime}\right), d$ is the distance (pc), and $P$ is the period of the orbit (yr); we obtain a value for $m_{1}+m_{2}$ of $1.20 \pm 0.25$ $\mathrm{M}_{\odot}$ [2]. Since our data also provide the semimajor axis of the absolute orbit of the main star, $a_{1}$ $\left({ }^{\prime \prime}\right)$, we can also calculate the masses of the individual components using the Kepler's Third Law in the form $\left(m_{2}^{3} /\left(m_{1}+m_{2}\right)^{2}=\left(a_{1} \cdot d\right)^{3} / P^{2}\right)$. We have preliminary values of the individual masses of HD 160934, that are $\sim 0.7 \mathrm{M}_{\odot}$ for the component $\mathrm{A}$ and $\sim 0.5 \mathrm{M}_{\odot}$ for the component $\mathrm{c}$.

\section{Other stars of the AB Dor-MG}

With the same EVN array used for HD 160934, we observed another star of the AB Dor-MG, EK Draconis. This star is placed at a distance of $\sim 33 \mathrm{pc}$ [10] and is a long period binary, which components are EK Dra A/B [11]. We could only detect the component A in the first epoch of observations (with an upper bound to the radio emission of the B component of $0.05 \mathrm{mJy}$ ).

Moreover, we also have a complete study of the binary system AB Dor B, placed at a distance of $\sim 15 \mathrm{pc}$. The components of $\mathrm{AB}$ Dor $\mathrm{B}, \mathrm{AB}$ Dor $\mathrm{Ba} / \mathrm{Bb}$, are separated $\sim 50$ mas, and show strong 
radio emission. We observed this target with the Australian VLBI Network, the Long Baseline Array (LBA), at a frequency of $8.4 \mathrm{GHz}$. In this case, we found that the masses of the individual components are $0.28 \pm 0.05 \mathrm{M}_{\odot}$ for $\mathrm{AB}$ Dor $\mathrm{Ba}$ and $0.25 \pm 0.05 \mathrm{M}_{\odot}$ for $\mathrm{AB}$ Dor Bb (Azulay et al. 2015, submitted).

\section{Conclusions and future work}

We have presented some results of our VLA/VLBI program focused to determine dynamical masses for PMS stars using radio techniques. Specifically, we have obtained preliminary values for the components of HD 160934, $\sim 0.7 \mathrm{M}_{\odot}$ for HD $160934 \mathrm{~A}$ and $\sim 0.5 \mathrm{M}_{\odot}$ for HD $160934 \mathrm{c}$. These results will be useful to provide new observational data to calibrate stellar evolution models for PMS stars. We are continuing our EVN program with a similar study of other stars in the AB DorMG: PW Andromedae, and LO Pegasus.

\section{Acknowledgments}

This work has been partially supported by the Spanish MINECO projects AYA2009-13036-C02-02 and AYA2012-38491-C02-01 and by the Generalitat Valenciana projects PROMETEO/2009/104 and PROMETEOII/2014/057. The Long Baseline Array is part of the Australia Telescope National Facility which is funded by the Commonwealth of Australia for operation as a National Facility managed by CSIRO. The European VLBI Network is a joint facility of European, Chinese, South African and other radio astronomy institutes funded by their national research councils. This research is supported by the European Community Framework Programme 7, Advanced Radio Astronomy in Europe, grant agreement No. 283393. R.A. acknowledges the Max-Planck-Institute für Radioastronomie for its hospitality.

\section{References}

[1] Azulay, R., Guirado, J. C., Marcaide, J. M., \& Martí-Vidal, I. 2013, Highlights of Spanish Astrophysics VII, 643

[2] Azulay, R., Guirado, J. C., Marcaide, et al. 2014, A\&A, 561, A38

[3] Evans, T. M., Ireland, M. J., Kraus, A. L., et al. 2012, ApJ, 744, 120

[4] Fekel, F. C. 1997, PASP, 109, 514

[5] Guirado, J. C., Martí-Vidal, I., Marcaide, J. M., et al. 2006, A\&A, 446, 733

[6] Guirado, J. C., Marcaide, J. M., Martí-Vidal, I., et al. 2011, A\&A, 533, A106

[7] Hillenbrand, L. A., \& White, R. J. 2004, ApJ, 604, 741

[8] Hormuth, F., Brandner, W., Hippler, S., et al. 2007, A\&A, 463, 707

[9] Janson, M., Brandner, W., Lenzen, R., et al. 2007, A\&A, 462, 615

[10] Järvinen, S. P., Berdyugina, S. V., \& Strassmeier, K. G. 2005, A\&A, 440, 735

[11] König, B., Guenther, E. W., Woitas, J., \& Hatzes, A. P. 2005, A\&A, 435, 215

[12] Lafrenière, D., Doyon, R., Marois, C., et al. 2007, ApJ, 670, 1367

[13] Lowrance, P. J., Becklin, E. E., Schneider, G., et al. 2005, AJ, 130, 1845 
[14] Mathieu, R. D., Baraffe, I., Simon, M., et al. 2007, Protostars and Planets V, 411

[15] Stassun, K. G., Mathieu, R. D., Vaz, L. P. R., et al. 2004, ApJS, 151, 357

[16] Torres, C. A. O., Quast, G. R., Melo, C. H. F., \& Sterzik, M. F. 2008, Handbook of Star Forming Regions, Volume II, 757

[17] Zuckerman, B., \& Song, I. 2004, ARA\&A, 42, 685 